\title{
Establishment of a Novel Simplified Surgical Model of Acute Liver Failure in the Cynomolgus Monkey
}

\author{
Lei Cai, ${ }^{1}$ Jun Weng, ${ }^{1}$ Lei Feng, ${ }^{1}$ Guolin He, ${ }^{1}$ Jiasheng Qin, ${ }^{1}$ Zhi Zhang, ${ }^{1}$ Yang Li, \\ Qing Peng, ${ }^{2}$ Zesheng Jiang, ${ }^{1}$ Mingxin Pan, ${ }^{1}$ and Yi Gao ${ }^{1,2}$ \\ ${ }^{1}$ Second Department of Hepatobiliary Surgery, Zhujiang Hospital, State Key Laboratory of Organ Failure Research, \\ Co-Innovation Center for Organ Failure Research, Southern Medical University, Guangzhou 510280, China \\ ${ }^{2}$ Institute of Regenerative Medicine, Zhujiang Hospital, Guangdong Provincial Research Center for \\ Artificial Organ and Tissue Engineering, Southern Medical University, Guangzhou 510280, China
}

Correspondence should be addressed to Yi Gao; drgaoy@126.com

Received 29 September 2016; Revised 19 November 2016; Accepted 28 November 2016

Academic Editor: Hartmut Jaeschke

Copyright (C) 2016 Lei Cai et al. This is an open access article distributed under the Creative Commons Attribution License, which permits unrestricted use, distribution, and reproduction in any medium, provided the original work is properly cited.

\begin{abstract}
Models using large animals that are suitable for studying artificial liver support system (ALSS) are urgently needed. Presently available acute liver failure (ALF) models mainly involve pigs or dogs. Establishment of current surgical ALF models (hepatectomy/devascularization) requires either very good surgical skills or multistep processes-even multiple stages of surgery. Therefore, it is necessary to develop a simplified surgical method. Here we report a novel simplified surgical ALF model using cynomolgus monkeys. Six monkeys underwent portal-right renal venous shunt combined with common bile duct ligation and transection (PRRS + CBDLT). Postoperatively, the monkeys had progressively increased listlessness, loss of appetite, and obvious jaundice. Blood biochemistry levels (Amm, ALT, AST, TBiL, DBiL, ALP, LDH, CK, and Cr) and prothrombin time (PT) were significantly increased (all $P<0.01)$ and albumin (ALB) was markedly reduced $(P<0.01)$ compared with baseline values. Histological examination of liver specimens on postoperative day 10 revealed cholestasis and inflammation. PRRS + CBDLT produced ALF that closely correlated with clinical situations. Compared with other surgical or drug ALF models, ours was simplified and animals were hemodynamically stable. This model could provide a good platform for further research on ALSS, especially regarding their detoxification functions.
\end{abstract}

\section{Introduction}

Acute liver failure (ALF) is a serious clinical disease known for its aggressive progression and poor prognosis. Although significant treatment improvements have been achieved in recent years, its mortality is still very high $[1,2]$. Liver transplantation is an effective therapeutic approach for ALF. However, its application has been limited in clinical practice for various reasons, including lack of organ availability, high cost, high technical requirements, and immunological rejection $[3,4]$. An artificial liver support system (ALSS) could be used as a bridge between liver failure and liver transplantation [5]. Before ALSS goes into clinical trials, establishment of an ideal experimental model in a large animal is desperately needed to test the feasibility, functionality, and safety $[6,7]$.
Currently, most available ALF models can be divided into two main categories: hepatotoxic drug models and surgical models. Hepatotoxic drug models, also termed chemical models, are frequently used models characterized by their simplicity and convenience. However, there are some limitations of such models, including poor reproducibility and potential risks and hazards for experimental personnel. In addition, large animals, compared with small animals, usually need relatively larger doses of drug, which leads to a rise in expenses. Of all surgical models, the total hepatectomy model (i.e., anhepatic model) is now rarely used due to the potential irreversibility, narrow window of opportunity, and high technical requirements [8]. The partial hepatectomy model, usually also involving devascularization, is seldom used because currently there is no unified standard for the volume of removed liver [9]. Ischemic models (complete/transient 
devascularization) are the most commonly used surgical models at present $[10,11]$, usually involving a portacaval shunt followed by hepatic artery occlusion and, in many situations, also requiring devascularization of accessory hepatic vessels and occlusion of the common bile duct $[9,11]$. In order to control hepatic ischemic time and prolong survival time of the experimental animals, surgical procedures to create this model normally are performed in two or three steps [12]. Such staging not only is complicated but also results in more injury to the animal. More importantly, excellent surgical skills are required to complete the portacaval shunt in the shortest possible time, which, in most cases, may be difficult for junior or early-career surgeons. Therefore, it is necessary to develop a simplified surgical model.

At present, some large animal models of ALF, such as those using pigs or dogs, have been reported [13-16]. However, there are few reports about model of ALF in nonhuman primates currently, especially regarding establishment of a surgical model. Due to the relatively close phylogenetic relationship to human beings, we chose the cynomolgus monkey for preclinical research regarding ALSS [17].

In this study, we established a novel simplified surgical model of ALF in the cynomolgus monkey, which may offer a good platform for further research into development of ALSS, especially regarding the detoxification function, which could provide temporary support for the insufficient or failing liver.

\section{Materials and Methods}

Six healthy male cynomolgus monkeys aged 7 to 11 years, weighing $8-12 \mathrm{~kg}$, were purchased from Guangdong Landao Biological Technology Co. Ltd. (number 33, Guanghua Road, Huangpu District, Guangzhou, Guangdong, China) (Certificate of Conformity SCXK [Guangdong] 2014-0010). The monkeys were housed individually in special stainless steel cages. An ongoing controlled environment was provided. All animal treatment procedures used in these experiments were performed in conformity with the "Regulations for the Administration of Affairs Concerning Experimental Animals (revised version)" released by the Ministry of Science and Technology of China in 2011. All experimental animals were cared for in accordance with standard guidelines from the Laboratory Animal Management Association of China. The experimental program was reviewed and approved by the Experimental Animal Institute of Zhujiang Hospital, Southern Medical University, Guangzhou, China (number ZJYY-2015-GDEK-001).

\subsection{Surgical Procedures}

2.1.1. Anesthesia and General Care. All operative procedures were performed at the animal experiment center of Guangdong Landao Biological Technology Co. Ltd. (number 33, Guanghua Road, Huangpu District, Guangzhou, Guangdong, China). All six monkeys were fasted for $12 \mathrm{~h}$ with free access to water before the experiment. Anesthesia was induced by an intramuscular injection of Zoletil 50 (tiletamine $125 \mathrm{mg}$ and zolazepam $125 \mathrm{mg}$; Virbac, France) at a dose of $15 \mathrm{mg} / \mathrm{kg}$ body weight, followed by atropine, $0.5 \mathrm{mg} / \mathrm{kg}$. The anesthetized animal was placed supinely on the operating table equipped with a warming blanket. After peroral endotracheal intubation, the animals maintained spontaneous breathing by continuous inhalation of isoflurane (1\%-2\%) and $\mathrm{O}_{2}(2 \mathrm{~L} / \mathrm{min})$.

Under this initial anesthesia regimen, electrodes were attached to the monkey to monitor heart rate (HR), blood pressure (BP) and oxygen saturation $\left(\mathrm{SpO}_{2}\right)$. The external jugular vein was punctured to connect a venous pressure meter to monitor the central venous pressure (CVP) and for intraoperative and postoperative intravenous infusions. Catheterization of the bladder was performed to monitor urine output.

2.1.2. Surgery. Surgical procedures were carried out under fully aseptic conditions. After a midline laparotomy was performed, a $5 \mathrm{~mm}^{3}$ sample of liver tissue was collected for histological examinations, as the control tissue. Firstly, the porta hepatis was exposed and the common bile duct, portal vein, and hepatic artery were all identified. Then the common bile duct was dissected and separated (Figure 1(a)), and, after a double ligation (with $2 / 0$ silk thread), it was transected. The portal vein was then freed to its bifurcation of left and right branches and confluence of the splenic vein. The right renal hilum was identified and then the right renal vein, right renal artery, and right ureter were exposed (Figure 1(b)). The right renal artery was doubly ligated but not transected. The right renal vein cephalad toward the renal hilum was ligated and then a lateral forceps was placed on the confluence of right renal vein and inferior vena cava. Using microsurgical scissors, a small cannula incision (about $1 / 3-1 / 2$ of the venous diameter) was made as close as possible to the former ligation of the right renal vein, and then a PVC-gel catheter, about $11 \mathrm{~cm} \times 0.5 \mathrm{~cm}$ (derived from a disposable sterile blood transfusion set) (Figure $1(\mathrm{c})$ ), containing $125 \mathrm{U} / \mathrm{mL}$ heparin saline, was inserted into the inferior vena cava at $0.5-1.0 \mathrm{~cm}$ depth from the incision. The catheter was adjusted to a good angle and fixed with $2 / 0$ silk thread. And, likewise, the portal vein was clamped at its confluence with the splenic vein and a ligation was carried out at the bifurcation of the portal vein. The other end of the catheter was clamped by a vascular clamp and was introduced into the portal vein away from the porta hepatis to a depth of $1.5-2.0 \mathrm{~cm}$. After proper placements were verified, the vascular clamp was removed and the catheter was opened. Thus a portal-right renal venous shunt combined with common bile duct ligation and transection was successfully completed (Figure 1(d)). After confirming that there was no obvious bleeding, the abdominal cavity was closed.

During the surgery, $100 \mathrm{~mL}$ of glucose and sodium chloride and cefazolin sodium pentahydrate $(60 \mathrm{mg} / \mathrm{kg})$ were infused. The anesthesia was discontinued at the end of surgery. The animals were kept in isolated cages and carefully monitored, and their behaviors were recorded. On postoperative day 10, all animals were euthanized. A necropsy was performed and specimens of liver were taken for histological examination.

2.2. Clinical Assessment. After the operation, the status of the animals (regarding mental status, appetite and activity, such 


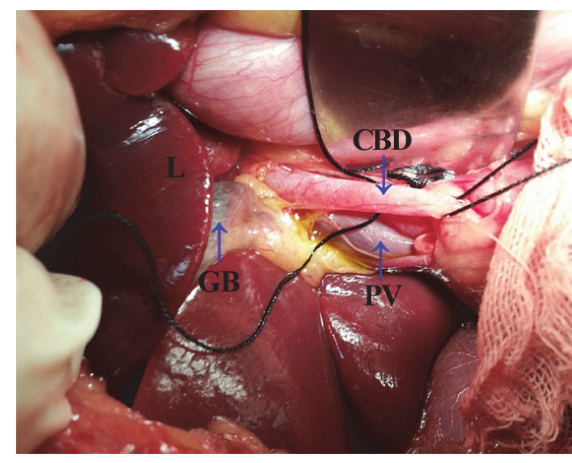

(a)

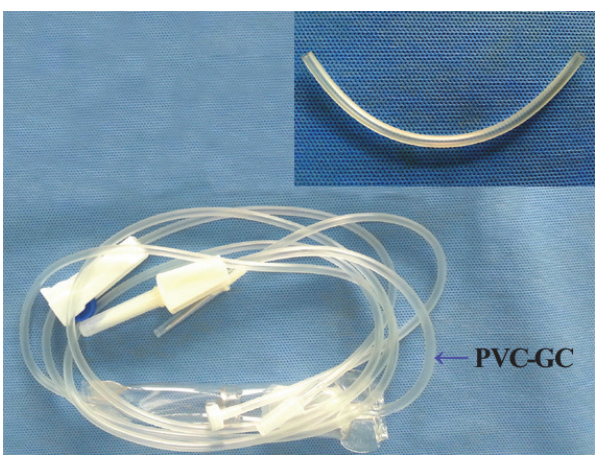

(c)

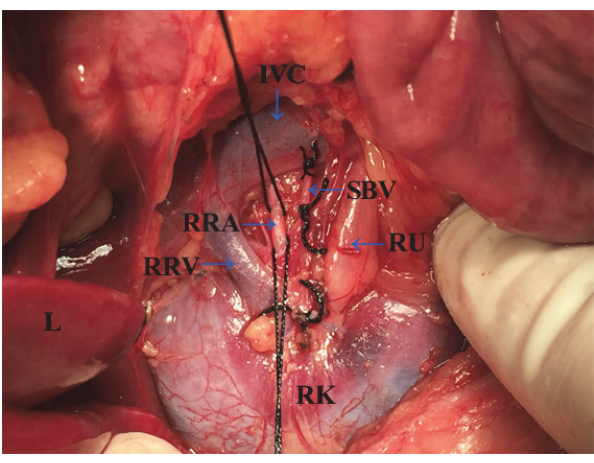

(b)

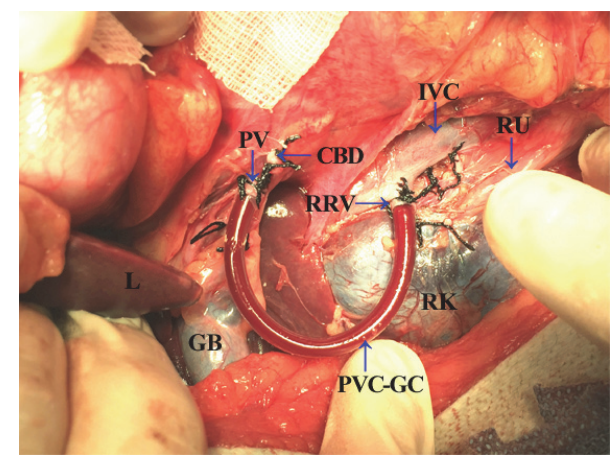

(d)

FIGURE 1: Simple surgical procedures for portal-right renal venous shunt combined with common bile duct ligation and transection. (a) Identification of the porta hepatis and dissection, ligation, and transection of common bile duct. (b) Identification of the right renal hilum and ligation of right renal artery. (c) PVC-gel catheter (about $11 \mathrm{~cm} \times 0.5 \mathrm{~cm}$ ) from a disposable sterile blood transfusion set. (d) Completion of right renal vein and portal vein intubation. RRV, right renal vein; RRA, right renal artery; RU, right ureter; SBV, small branch vein; IVC, inferior vena cava; CBD, common bile duct; PV, portal vein; L, Liver; RK, right kidney; GB, gall bladder; PVC-GC, PVC-gel catheter.

as ability to open eyes, to stand up, and to walk, and responses to visual, acoustic, and painful stimuli) was observed and recorded. Presence of vomiting and jaundice were also noted. All these assessments were made in order to look for signs of ALF and hepatic encephalopathy.

2.3. Assessment of Blood Biochemistry and Coagulation. Blood samples were collected at 8 time points, as follows: before the operation (baseline), at the end of the operation $(0 \mathrm{~h}), 8$ hours after the operation $(8 \mathrm{~h})$, postoperative day $1(1 \mathrm{~d})$, postoperative day $2(2 \mathrm{~d})$, postoperative day $3(3 \mathrm{~d})$, postoperative day $7(7 \mathrm{~d})$, and postoperative day $10(10 \mathrm{~d})$. The following blood levels were measured: ammonia (Amm), alanine aminotransferase (ALT), aspartate aminotransferase (AST), albumin (ALB), total bilirubin (TBiL), direct bilirubin (DBiL), alkaline phosphatase (ALP), lactate dehydrogenase (LDH), creatine phosphate kinase (CK), blood urea nitrogen $(\mathrm{BUN})$, and creatinine $(\mathrm{Cr})$. In addition, the prothrombin time (PT) was determined.

2.4. Histological Examination. On postoperative day 10, all animals were euthanized and specimens of liver were obtained for pathological and immunohistochemical examination. Liver tissues were trimmed into $5 \mathrm{~mm}^{3}$ blocks, fixed with $10 \%$ formaldehyde, and embedded in paraffin after dehydration. After sectioning, hematoxylin-eosin staining, terminal deoxynucleotidyl transferase-mediated dUTP nickend labeling (TUNEL) staining, Masson trichrome staining, and Ki-67 immunohistochemistry staining were performed to assess the extent of hepatic pathological changes, hepatocyte apoptosis, hepatic fibrosis, and hepatocyte proliferation, respectively.

2.5. Statistical Analysis. Numerical data were displayed as mean \pm standard deviation (SD). All data were analyzed with GraphPad Prism 5 software (GraphPad Software Inc., San Diego, CA, USA) by two-tailed Student's $t$-test. Differences having $P$ values of $<0.05$ were considered statistically significant.

\section{Results}

3.1. Surgical Outcomes. All the experimental monkeys survived for more than 10 days. This and other surgical performance-related data are shown in Table 1. Changes of heart rate (HR), blood pressure (BP), central venous pressure (CVP), and oxygen saturation $\left(\mathrm{SpO}_{2}\right)$ in animals before, during (at the catheterization of the portal vein and the catheterization of the right renal venous), and after the operation are shown in Figures 2(a)-2(d). As can be seen, HR, $\mathrm{BP}$, and CVP were stable throughout the procedure except 


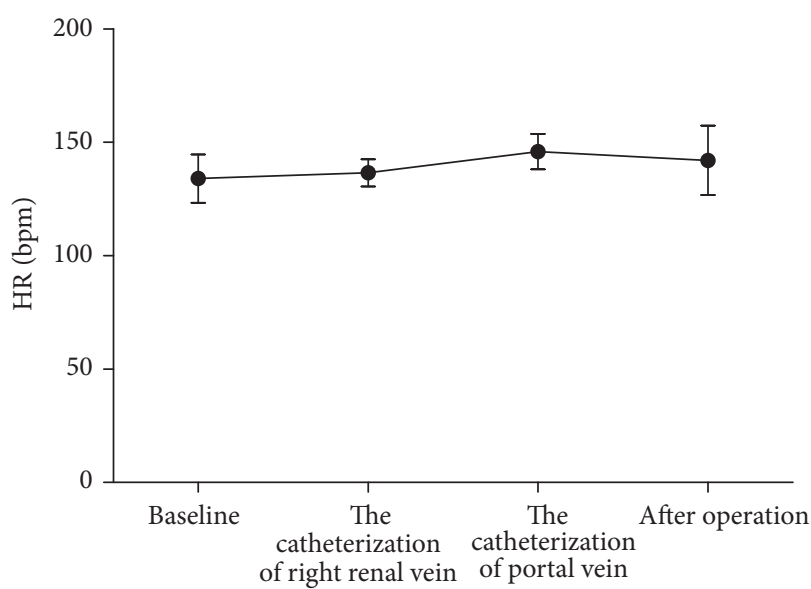

(a)

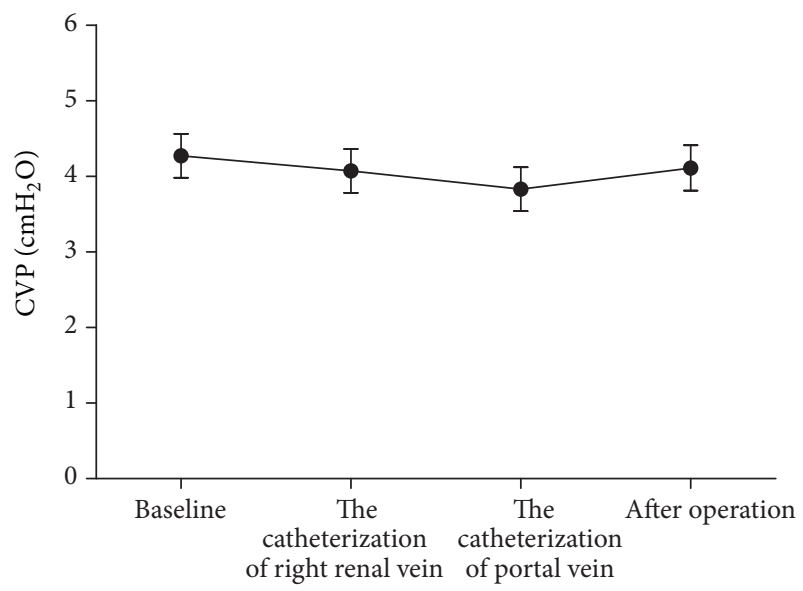

(c)

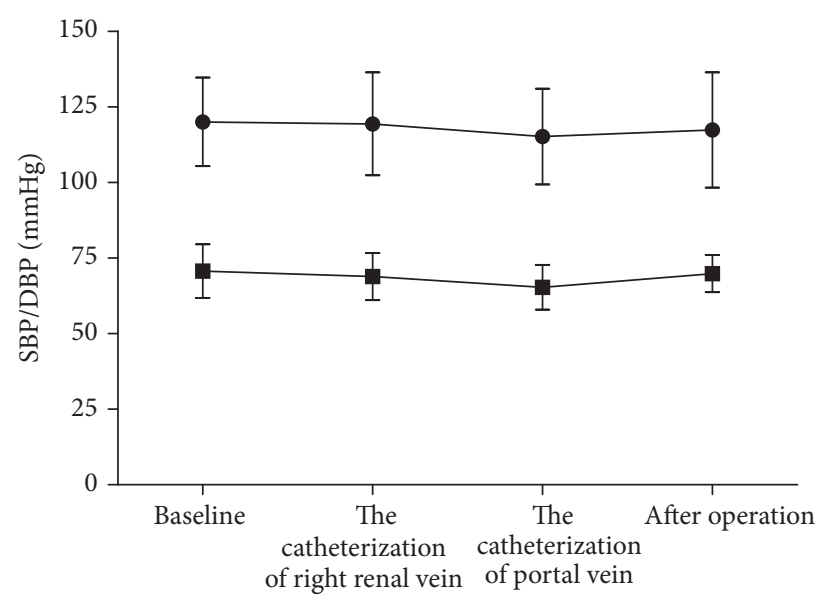

SBP

DBP

(b)

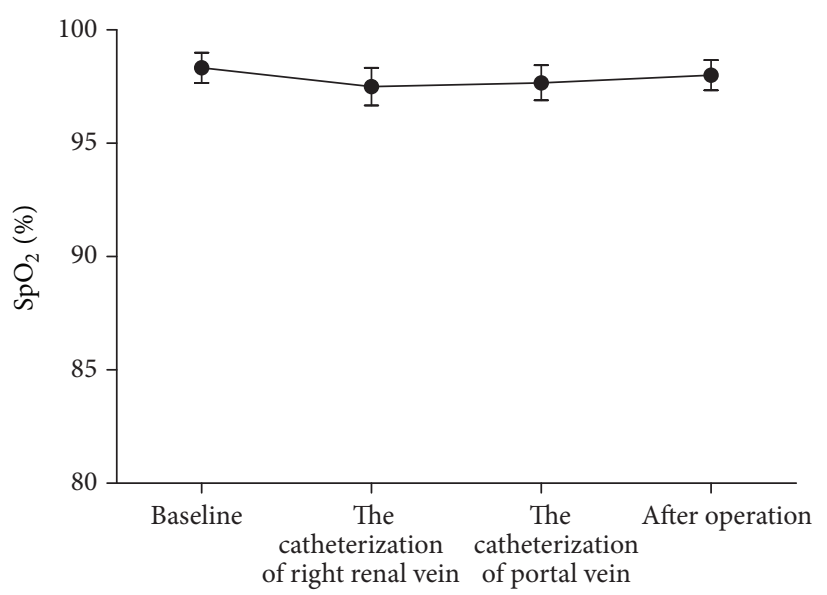

(d)

FIGURE 2: Changes of HR (a), BP (SBP/DBP) (b), CVP (c), and $\mathrm{SpO}_{2}$ (d) during surgery. Mean \pm SD, $n=6$. HR: heart rate; SBP: systolic blood pressure; DBP: diastolic blood pressure; CVP: central venous pressure; $\mathrm{SpO}_{2}$ : oxygen saturation.

TABLE 1: Basic characteristics of PRRS + CBDLT in monkeys.

\begin{tabular}{lc}
\hline Items & Results \\
\hline Operation time $(\mathrm{min})$ & $36 \pm 4$ \\
Operation ABL $(\mathrm{mL})$ & $25 \pm 3$ \\
Operation ABT $(\mathrm{mL})$ & 0 \\
Port vein occlusion time (min) & $5 \pm 2$ \\
10 -d survival & $100 \%(6 / 6)$ \\
\hline
\end{tabular}

Mean $\pm \mathrm{SD}, n=6$.

PRRS + CBDLT, portal-right renal venous shunt combined with common bile duct ligation and transection; ABL, amount of blood loss; ABT, amount of blood transfusion; 10-d, 10 days.

for slight fluctuations during the clamping of the portal vein. $\mathrm{SpO}_{2}$ was consistently maintained above $95 \%$.

3.2. Clinical Assessment. After the operation, all the monkeys became increasingly listless and apathetic, as well as developing a dull reaction to visual, acoustic, and painful stimuli, even though they could open their eyes, get up, and walk. Vomiting appeared in 6 animals at postoperative days $1,3,4,4,7$, and 10. Mental indifference and drowsiness, but no coma, were observed in 4 animals after the operation. On postoperative day 10, all monkeys were alive and had obvious jaundice. Hepatic coma appeared in 1 animal on postoperative day 3 , and in another on postoperative day 8 , but subsided after $6 \mathrm{~h}$ and $12 \mathrm{~h}$, respectively.

3.3. Assessment of Blood Biochemistry and Coagulation. Amm, TBiL, DBiL, and ALP levels progressively increased after the operation. PT, AST, ALT, CK, and LDH were also significantly increased postoperatively compared with baseline levels, especially in the first 3 days after surgery. The serum ALB level significantly decreased postoperatively and reached its lowest level on postoperative day 7. The levels of BUN and $\mathrm{Cr}$ were slightly higher than those before the operation but returned almost to baseline levels on postoperative day 7 (Figures 3(a)-3(i)). On postoperative day 10, the increase of Amm, TBiL, DBiL, AST, ALT, ALP, CK, LDH, Cr, and PT and 


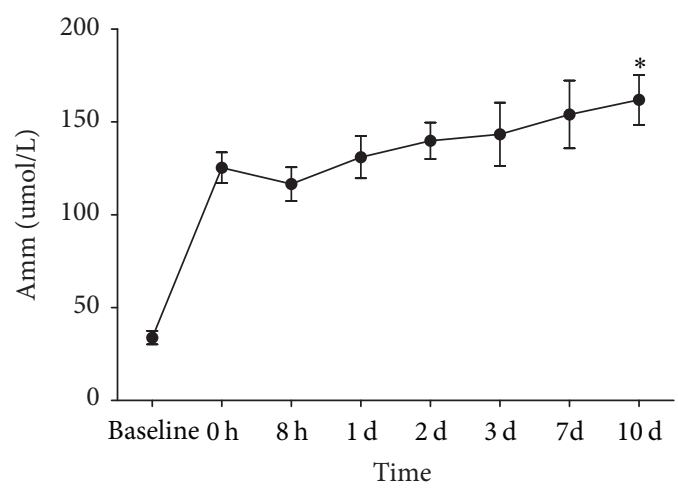

(a)

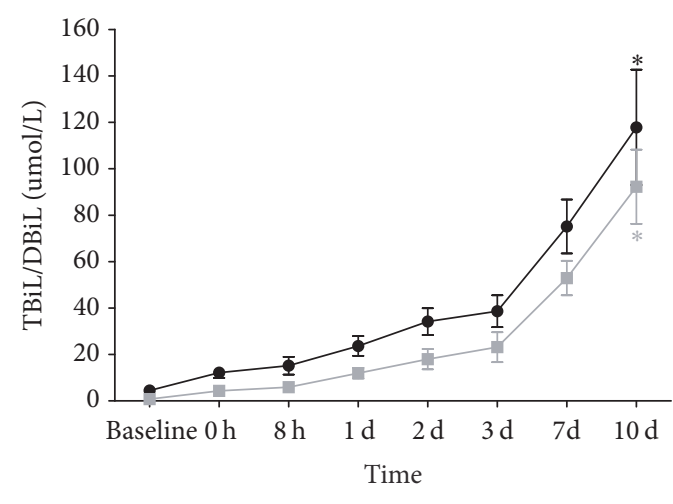

$\rightarrow$ TBiL

$\rightarrow$ DBiL

(c)

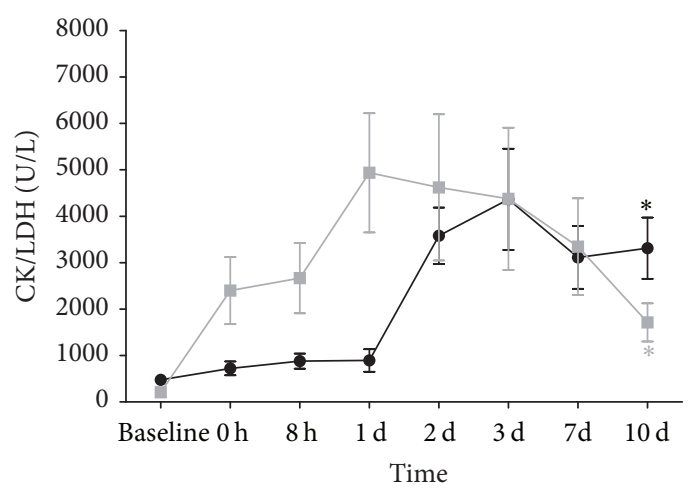

- $\mathrm{CK}$

$-\mathrm{LDH}$

(e)

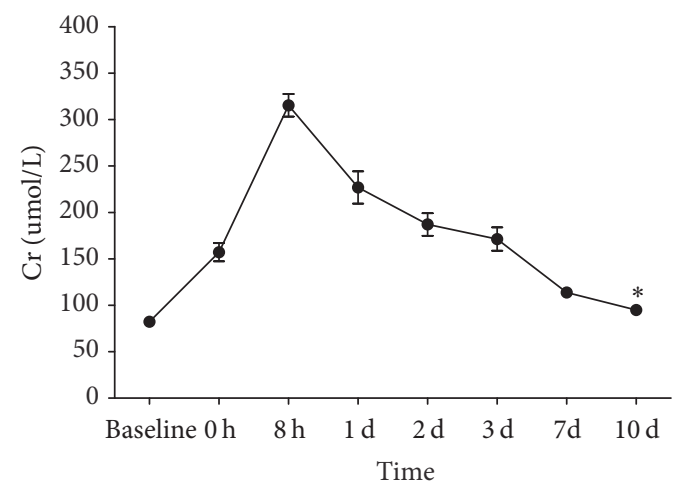

(g)

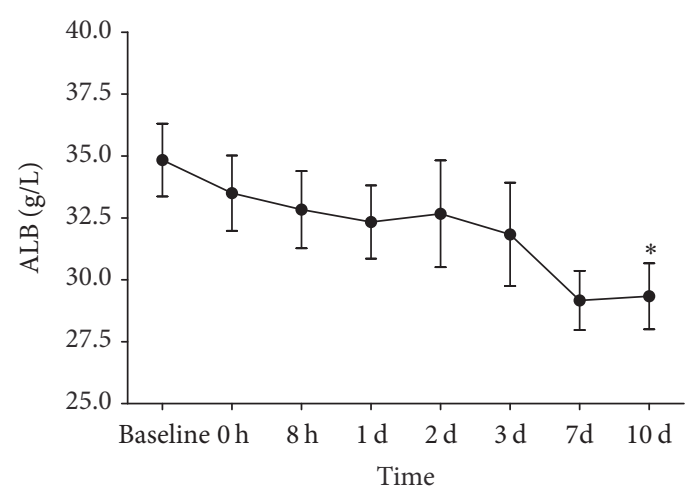

(b)

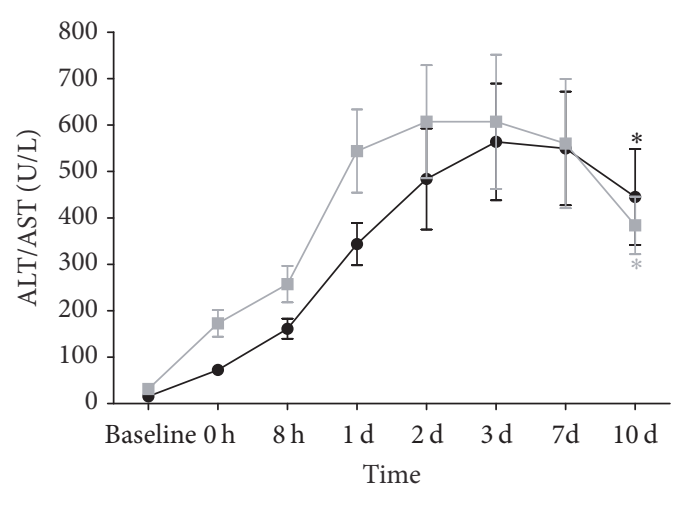

$\rightarrow$ ALT

$\rightarrow$ AST

(d)

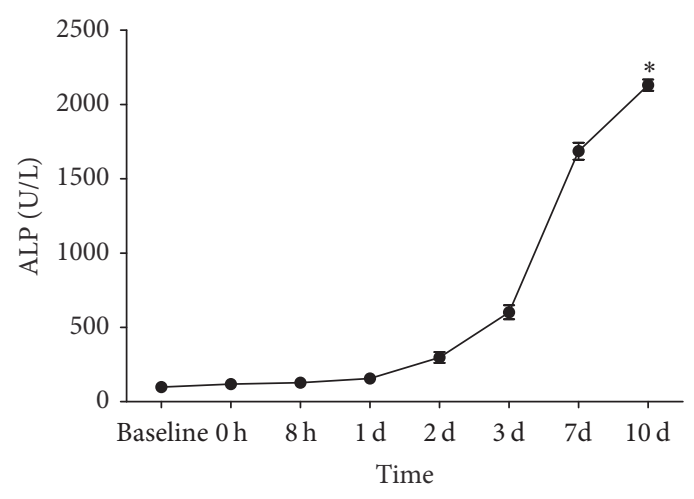

(f)

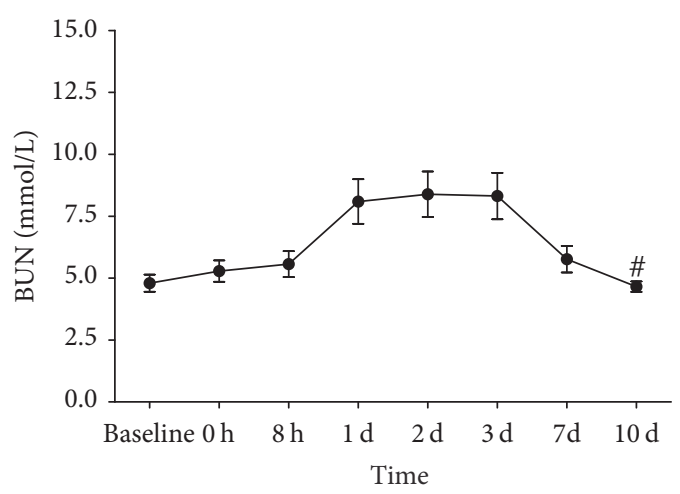

(h)

Figure 3: Continued. 


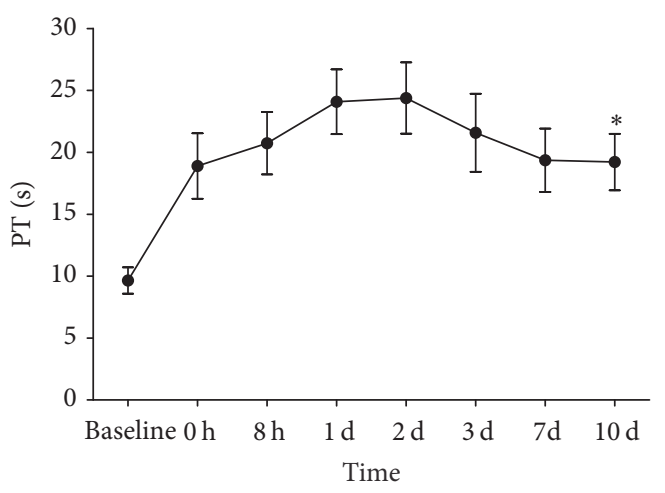

(i)

Figure 3: Changes in levels of Amm (a), ALB (b), TBiL (c), DBiL (c), ALT (d), AST (d), CK (e), LDH (e), ALP (f), Cr (g), BUN (h), and PT (i) prior to operation (baseline) and at $0 \mathrm{~h}, 8 \mathrm{~h}, 1 \mathrm{~d}, 2 \mathrm{~d}, 3 \mathrm{~d}, 7 \mathrm{~d}$, and $10 \mathrm{~d}$ after operation. Mean $\pm \mathrm{SD} .{ }^{*} P<0.01$ versus baseline, ${ }^{\#} P>0.05$ versus baseline. Amm: ammonia; ALB: albumin; TBiL: total bilirubin; DBiL: direct bilirubin; ALT: alanine aminotransferase; AST: aspartate aminotransferase; CK: creatine phosphate kinase; LDH: lactate dehydrogenase; ALP: alkaline phosphatase; Cr: creatinine; BUN: blood urea nitrogen; PT: prothrombin time.

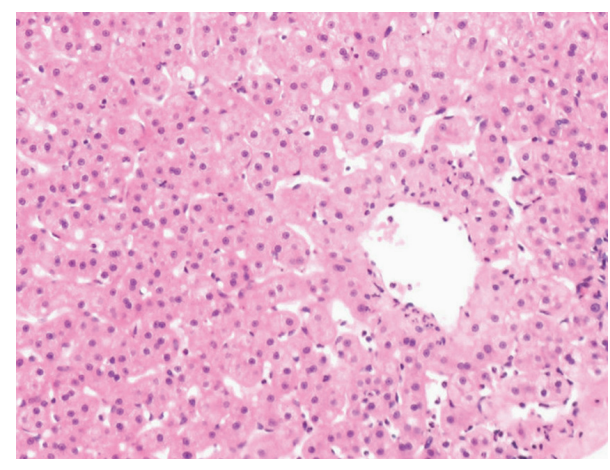

(a)

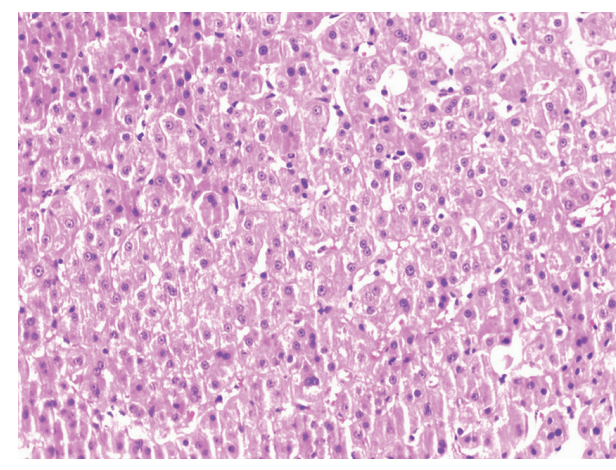

(b)

FIGURE 4: Pathological hepatic features of experimental monkey. (a) Representative preoperative specimen. (b) Representative specimen from postoperative day 10 . Hematoxylin-eosin stain, $\times 20$.

the decrease of ALB were all significant statistically, compared with baseline levels $(P<0.01)$. However, no difference in BUN between postoperative day 10 and the baseline value was found $(P>0.05)$ (Figures 3(a)-3(i)).

3.4. Histological Examination. The autopsies revealed that all the monkeys had intrahepatic cholestasis and extrahepatic bile duct obstruction due to marked dilatation of the proximal bile ducts and gallbladder. Pathological examination of liver specimens from postoperative day 10 revealed denaturation of hepatocytes and structural damage of hepatocyte architecture (Figure 4(b)), compared with preoperative specimens, which contained clear hepatocytes and normal hepatic sinusoid architecture (Figure 4(a)). In terms of TUNEL staining, positive cells were not seen in the preoperative liver specimens (Figure 5(a)). However, a number of apoptotic cells were detected after the surgery (Figure 5(b)). Masson trichrome staining revealed no obvious differences between preoperative and postoperative liver specimens (Figures 6(a) and 6(b)), which indicated that hepatic fibrosis had not formed in such a short span of time. Ki-67 staining was negative in preoperative liver sections, whereas some Ki67-positive hepatocytes appeared in the liver tissue from postoperative day 10 (Figures $7(\mathrm{a})$ and $7(\mathrm{~b})$ ).

\section{Discussion}

An appropriate large animal model that closely correlates with usual clinical situations is urgently needed to assess the safety and efficacy of ALSS. The criteria for an ideal animal model were outlined by Terblanche and Hickman in 1991, which prevailed and have been further supplemented [18]. At present, the widely accepted criteria for a satisfactory ALF animal model include the following: (i) potential reversibility, (ii) reproducibility, (iii) liver specific toxic damage, (iv) large animal, (v) suitable window of opportunity, (vi) ethical practices, (vii) harm minimization to experimenter and environment, (viii) physiological metabolism similar to human, and (ix) change of consciousness.

A lot of research has been done on how to obtain a satisfactory model using a simple approach. However, current models have various deficiencies and limitations. 


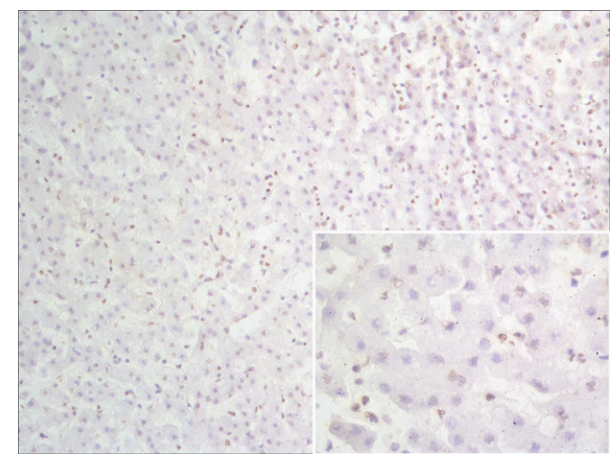

(a)

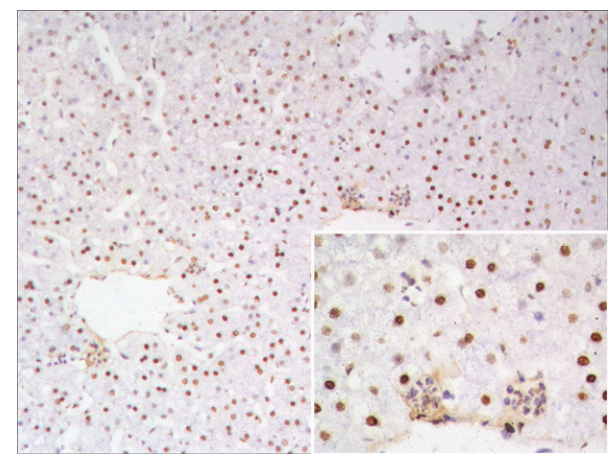

(b)

FIgURE 5: TUNEL staining in liver of the experimental monkey. (a) Preoperative liver section showed no evidence of apoptosis ( $\times 10)$. (b) A number of apoptotic cells were detected in the liver section from postoperative day $10(\times 10)$. High magnification $(\times 20)$ images are shown in the lower right corner.

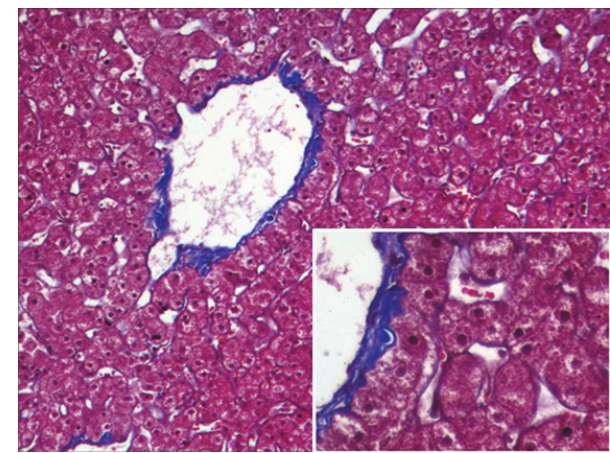

(a)

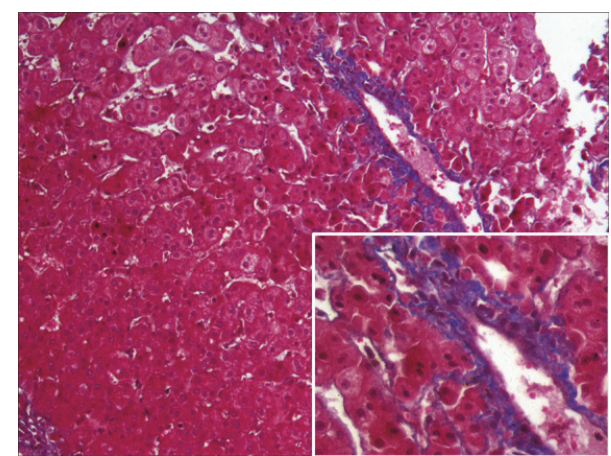

(b)

FIGURE 6: Masson trichrome staining in liver of the experimental monkey. Fibrotic change did not appear in preoperative liver section (a) and liver section from postoperative day 10 (b) $(\times 10)$. High magnification $(\times 20)$ images are shown in the lower right corner.

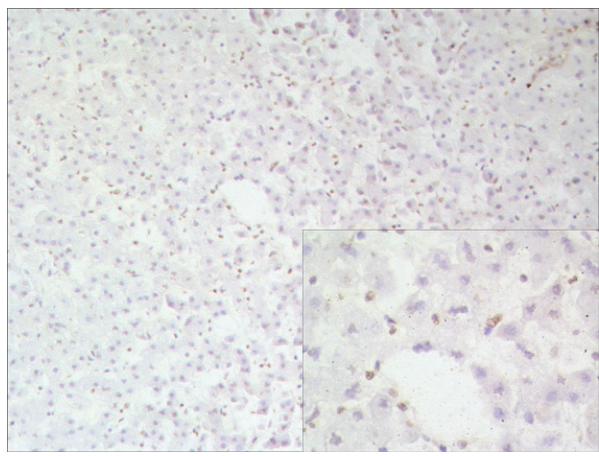

(a)

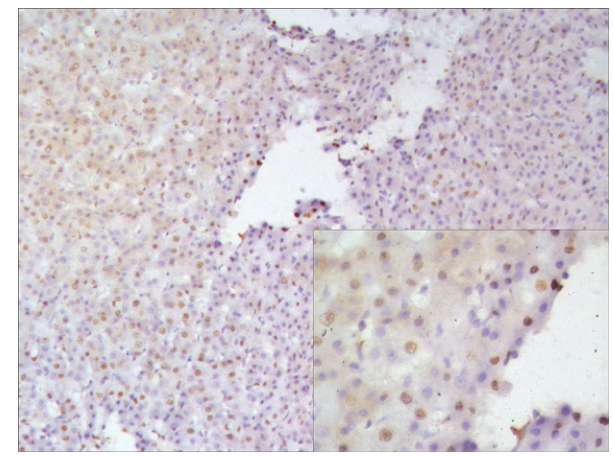

(b)

Figure 7: Ki-67 staining of proliferating hepatocytes in liver of the experimental monkey. (a) Preoperative liver section showed a negative expression. (b) Liver section from postoperative day 10 revealed a few positively stained nuclei $(\times 10)$. High magnification $(\times 20)$ images are shown in the lower right corner.

Animal models of ALF available presently mainly include two broad types: hepatotoxic drug models and surgical models.

Many different drug models (e.g., those created using Dgalactosamine, acetaminophen, carbon tetrachloride, amanitin, concanavalin A, lipopolysaccharide, thioacetamide, and nitrosamines) have been described in the literature [18-23]. The greatest advantage of drug models is that no anesthetics and complex operations are required. However, there are also some drawbacks to this type of model. First of all, heterogeneous results and poor reproducibility frequently occur, both clinically and biochemically, in these models 
because the ability of the liver to metabolize drugs is very different between species and according to age. Secondly, most of the toxic drugs not only are hepatotoxic but also have nephrotoxicity, cardiotoxicity, other organ damage, or serious adverse effects. Thirdly, costs would be greatly increased due to the increased dosages of some agents required for large animals. And, lastly, some toxins pose high risks for laboratory personnel. Therefore, toxic drug models need to be further improved despite inclusion of a simple procedure.

Compared with hepatotoxic drug models, surgical models are preferred increasingly by some researchers, particularly in large animals $[24,25]$. Generally, the primary surgical procedures used for modeling could be classified into three major categories: devascularization (total and partial), hepatectomy (total and partial), and a combination of both $[26,27]$. The total hepatectomy model, which is also called the anhepatic model, inevitably produces ALF, so it is considered a pure model [28]. However, there are still many defects of this model, such as the potential irreversibility, a narrow window of opportunity and the requirement of great surgical skill. Moreover, this kind of model produces a completely different clinicopathologic entity in which there is continuous, massive toxin release from damaged cells into the bloodstream [29]. All these factors led to a gradual decline in the use of the anhepatic model. In recent years, partial hepatectomy is gradually substituting for total hepatectomy and has become the prevailing approach to hepatectomy models [26, 27, 30]. A high level of professional competence is necessary for creating these models. The difficulty is that liver regeneration should be induced while adequate resection causes ALF. But, unfortunately, there is currently no unified standard regarding the liver volume to be removed, which is critical for this model type. So, complexity, to a certain extent, becomes an obvious drawback of this approach. Nevertheless, this kind of model has been applied successfully to evaluate ALSS [20], and in recent years it is being used increasingly to study liver transplantation and liver regeneration.

Total (complete) liver devascularization usually involves portacaval shunt together with hepatic artery blockage, and, in many situations, devascularization of the gastric and diaphragmatic branches and ligation of the common bile duct are needed $[11,31]$. The animals in these models usually died rapidly due to irreversibility. Partial (transient) liver devascularization usually refers to portacaval shunt combined with temporary hepatic artery occlusion [32]. This model is more or less reversible depending on how long the hepatic artery is blocked. According to previous reports, the mortality was 50\% and 90\%, respectively, after 4 and 6 hours of clamping [33]. In order to control the length of hepatic ischemia and prolong the survival time of model animals, partial devascularization procedures normally need two or three steps $[32,34]$, making the surgery more complicated and traumatic for animals. Therefore, it is very necessary to develop modified or improved surgical procedures to produce an ALF model, although partial ischemic models have been developed using a variety of species [15, 34].

We previously reported an irreversible porcine ALF model that was created using two-phase surgery. Although the animal model was used subsequently to study ALSS, there are still some deficiencies to be improved, such as the complexity of the operation, the large degree of trauma for the animals, and the narrow window of opportunity for therapy (survival time was $13.17 \pm 1.47 \mathrm{~h}$ ) [35].

In the current study, we chose portal-right renal venous shunting combined with common bile duct ligation and transection (PRRS + CBDLT) as our modeling approach. On the one hand, the portal-right renal venous shunt brought about a decrease of blood flowing into the liver and liver atrophy, which led to further liver damage and liver dysfunction. In addition, hepatic encephalopathy could be induced by this portacaval shunt, which could be explained by the increased level of blood ammonia. On the other hand, the obstruction of the common bile duct and loss of bile excretory function caused by common bile duct ligation and transection resulted in an accumulation of bilirubin. Subsequently, hepatotoxicity and inflammation gave rise to ALF. Actually, development of improved blood biochemistry indices in the model animal is one of the main concerns in evaluation of ALSS, especially the detoxification function. If there is model in which there is a significant elevation of ammonia, total bilirubin, AST, and ALT (etc.) levels but without rapid death, it would be sufficient and useful to test the detoxification function of the ALSS. It is for this reason that we established our model using PRRS + CBDLT.

In the current study, we choose postoperative day 10 as our endpoint for the following reasons: (1) In our preexperiment, one animal survived for 27 days and ultimately died from severe hyperbilirubinemia without intervention and the other one survived more than one month. Animals that are awake, are neurologically stable, and do not experience rapid death are favorable for further research on ALSS. (2) Blood biochemistry levels (Amm, ALT, AST, TBiL, DBiL, ALP, LDH, $\mathrm{CK}$, and $\mathrm{Cr}$ ) and prothrombin time (PT) did not reach peak values simultaneously. (3) As mentioned previously, a sufficient rise in the toxins, such as Amm, TBiL, and DBiL, can satisfy the requirement for testing the detoxification function of the ALSS.

In our model, Amm, TBiL, DBiL, and ALP never increased to a critical level. But, on postoperative day 10 , they were remarkably high, at about 5-fold, 26-fold, 115-fold, and 21-fold, respectively, compared with preoperative data; they were all also statistically significantly higher compared with baseline levels $(P<0.01)$. Blood ALT, AST, and PT were also high and hypoalbuminemia was present in almost all monkeys on postoperative day 10. The histological assessment revealed inflammation with denaturation and necrosis of hepatocytes as well as loss of hepatocyte architecture, which also offered strong evidence for ALF.

The biggest difference between our model and other ischemic models was that we produced an occlusion of the portal vein but retained blood supply through the hepatic artery, in combination with biliary obstruction. The model established by our procedure has the following advantages: (1) The model was easy to reproduce because it involves simpler surgery compared with other approaches. Shunting was established by intubation instead of anastomosis between the portal vein and inferior vena cava, which lowered surgical difficulty and shortened the operation time (our intubation 
shunt procedure required only a few minutes). (2) This modeling procedure had a high success rate because venous return to the heart and other nonhepatic visceral organs were little influenced while achieving liver devascularization. Gastrointestinal venous stasis, unavoidable in portacaval shunting, was greatly alleviated due to obviously shortened operation time. In addition, cannulation was performed in the right renal vein prior to the portal vein, which also, to a certain degree, diminished the hemodynamic fluctuation of the portal vein. (3) Induction of liver ischemic tolerance resulting from partial hepatic inflow occlusion during the operation allowed our animals to survive for a longer time, which would provide a longer window of opportunity of conducting further research on ALSS.

We chose the cynomolgus monkey because it is a nonhuman primate with very similar structural, metabolic, biochemical, physiological, and immunological characteristics to human beings that has become recognized as an ideal experimental animal for establishing ALF models to provide a good platform for the preclinical development of ALSS $[17,36]$.

However, the model established in the current study procedure has some limitations. As the portal vein is ligated in this model, the possibility of testing the efficacy of hepatocyte or stem cell transplantation in ALF is excluded. In addition, the small sample size of six monkeys means that the results will have to be replicated in a larger study.

\section{Conclusions}

In conclusion, we established a novel surgical model for ALF in the cynomolgus monkey through portal-right renal venous shunt combined with common bile duct ligation and transection. As a single-setting procedure, the surgery is simplified and easy to reproduce and popularize, and the mortality rate of the model animals was low owing to hemodynamic stabilization and less trauma. This model may prove helpful for evaluating the safety and efficacy of various ALSS, especially regarding their detoxification functions.

\section{Competing Interests}

All authors of this article declare that they have no conflict of interests.

\section{Acknowledgments}

This work was co-supported by the Science and Technology Planning Project of Guangdong Province, China (no. 2013B091100001, no. 2014B020227002, no. 2015B090903069, and no. 2015B020229002), the National High Technology Research and Development Program of China (863 Programs, no. 2012AA020505), the Medical Scientific Research Foundation of Guangdong Province, China (no. C1036114), and the Interdisciplinary Innovation Team Cultivation Plan Project of Southern Medical University for "The Research and Development of Liver Tissue Engineering Biomaterial and Key Technology Related to Liver Failure Replacement
Therapy" (number 1023-1063, Shatai South Road, Baiyun District, Guangzhou, Guangdong, China). The authors thank Mr. Yuefeng Li and Mr. Xingwen Qin at the Department of Experimental Animals in Guangdong Landao Biological Technology Co. Ltd. (number 33, Guanghua Road, Huangpu District, Guangzhou, Guangdong, China) who kindly provided surgical tools, surgical assistance, blood sample collection, and animal care services.

\section{References}

[1] N. Akamatsu, Y. Sugawara, and N. Kokudo, "Acute liver failure and liver transplantation," Intractable \& Rare Diseases Research, vol. 2, no. 3, pp. 77-87, 2013.

[2] W. Bernal and J. Wendon, "Acute liver failure," The New England Journal of Medicine, vol. 369, no. 26, pp. 2525-2534, 2013.

[3] M. C. Donnelly, P. C. Hayes, and K. J. Simpson, “The changing face of liver transplantation for acute liver failure: assessment of current status and implications for future practice," Liver Transplantation, vol. 22, no. 4, pp. 527-535, 2016.

[4] J. P. Vacanti and K. M. Kulig, "Liver cell therapy and tissue engineering for transplantation," Seminars in Pediatric Surgery, vol. 23, no. 3, pp. 150-155, 2014.

[5] F. Saliba and D. Samuel, "Artificial liver support: a real step forward," Minerva Medica, vol. 106, no. 1, pp. 35-43, 2015.

[6] X.-L. Shi, Y. Gao, Y. Yan et al., "Improved survival of porcine acute liver failure by a bioartificial liver device implanted with induced human functional hepatocytes," Cell Research, vol. 26, no. 2, pp. 206-216, 2016.

[7] K. C. L. Lee, L. A. Baker, G. Stanzani et al., "Extracorporeal liver assist device to exchange albumin and remove endotoxin in acute liver failure: results of a pivotal pre-clinical study," Journal of Hepatology, vol. 63, no. 3, pp. 634-642, 2015.

[8] K. Thiel, M. Schenk, A. Etspüler et al., "A simple dummy liver assist device prolongs anhepatic survival in a porcine model of total hepatectomy by slight hypothermia," BMC Gastroenterology, vol. 11, article 79, 2011.

[9] M. J. Tuñón, M. Alvarez, J. M. Culebras, and J. GonzálezGallego, "An overview of animal models for investigating the pathogenesis and therapeutic strategies in acute hepatic failure," World Journal of Gastroenterology, vol. 15, no. 25, pp. 3086-3098, 2009.

[10] A. Tsaroucha, C. Chondrogiannis, A. Mani, and C. Staikou, "Myocardial involvement during ischemia-induced acute liver failure in the pig," Journal of Investigative Surgery, vol. 26, no. 2, pp. 99-104, 2013.

[11] A. Al-Chalabi, E. Matevossian, A.-K. V. Thaden et al., "Evaluation of the Hepa Wash ${ }^{\circledR}$ treatment in pigs with acute liver failure," BMC Gastroenterology, vol. 13, no. 1, article 83, 2013.

[12] K. Sawara, P. Desjardins, N. Chatauret, A. Kato, K. Suzuki, and R. F. Butterworth, "Alterations in expression of genes coding for proteins of the neurovascular unit in ischemic liver failure," Neurochemistry International, vol. 55, no. 1-3, pp. 119-123, 2009.

[13] R. G. Kristiansen, C. F. Rose, O.-M. Fuskevåg et al., "LOrnithine phenylacetate reduces ammonia in pigs with acute liver failure through phenylacetylglycine formation: a novel ammonia-lowering pathway," American Journal of PhysiologyGastrointestinal and Liver Physiology, vol. 307, no. 10, pp. G1024G1031, 2014.

[14] V. Sharma, G. A. M. ten Have, L. Ytrebo et al., "Nitric oxide and L-arginine metabolism in a devascularized porcine 
model of acute liver failure," American Journal of PhysiologyGastrointestinal and Liver Physiology, vol. 303, no. 3, pp. G435G441, 2012.

[15] Z. Chen and Y.-T. Ding, "Functional evaluation of a new bioartificial liver system in vivo and in vivo," World Journal of Gastroenterology, vol. 12, no. 8, pp. 1312-1316, 2006.

[16] S. S. Awad, M. R. Hemmila, O. S. Soldes et al., "A novel stable reproducible model of hepatic failure in canines," Journal of Surgical Research, vol. 94, no. 2, pp. 167-171, 2000.

[17] Z. Zhang, Y.-C. Zhao, Y. Cheng, G.-D. Jian, M.-X. Pan, and Y. Gao, "Hybrid bioartificial liver support in cynomolgus monkeys with D-galactosamine-induced acute liver failure," World Journal of Gastroenterology, vol. 20, no. 46, pp. 1739917406, 2014.

[18] M. Maes, M. Vinken, and H. Jaeschke, "Experimental models of hepatotoxicity related to acute liver failure," Toxicology and Applied Pharmacology, vol. 290, pp. 86-97, 2016.

[19] Z. Q. Qiu, L. Cai, and D. S. Chen, "Total flavonoids from mimosa pudica protects carbon tetrachloride-induced acute liver injury in mice," Journal of International Translational Medicine, vol. 3 , no. 1, pp. 6-10, 2015.

[20] L. A. Baker, K. C. Lee, C. P. Jimenez et al., "Circulating microRNAs reveal time course of organ injury in a porcine model of acetaminophen-induced acute liver failure," PLoS ONE, vol. 10, no. 5, Article ID e0128076, 2015.

[21] K. C. L. Lee, C. P. Jimenez, H. Alibhai et al., "A reproducible, clinically relevant, intensively managed, pig model of acute liver failure for testing of therapies aimed to prolong survival," Liver International, vol. 33, no. 4, pp. 544-551, 2013.

[22] J. M. Glorioso, S. A. Mao, B. Rodysill et al., "Pivotal preclinical trial of the spheroid reservoir bioartificial liver," Journal of Hepatology, vol. 63, no. 2, pp. 388-398, 2015.

[23] N. Zhou, J. Li, Y. Zhang et al., "Efficacy of coupled low-volume plasma exchange with plasma filtration adsorption in treating pigs with acute liver failure: a randomised study," Journal of Hepatology, vol. 63, no. 2, pp. 378-387, 2015.

[24] J. Prazak, E. Laszikova, T. Pantoflicek, O. Ryska, E. Koblihova, and M. Ryska, "Cerebral microdialysis reflects the neuroprotective effect of fractionated plasma separation and adsorption in acute liver failure better and earlier than intracranial pressure: a controlled study in pigs," BMC Gastroenterology, vol. 13, no. 1, article 98, 2013.

[25] O. Ryska, T. Pantoflicek, E. Laszikova, J. Prazak, E. Koblihova, and M. Ryska, "Artificial liver support system reduces intracranial pressure more effectively than bioartificial system: an experimental study," International Journal of Artificial Organs, vol. 35, no. 7, pp. 503-510, 2012.

[26] N. Papoutsidakis, N. Arkadopoulos, V. Smyrniotis et al., "Early myocardial injury is an integral component of experimental acute liver failure-a study in two porcine models," Archives of Medical Science, vol. 7, no. 2, pp. 217-223, 2011.

[27] O. Detry, Y. Gaspar, J.-P. Cheramy-Bien, P. Drion, M. Meurisse, and J.-O. Defraigne, "A modified surgical model of fulminant hepatic failure in the rat," Journal of Surgical Research, vol. 181, no. 1, pp. 85-90, 2013.

[28] K. Knubben, C. Thiel, M. Schenk et al., "A new surgical model for hepatectomy in pigs," European Surgical Research, vol. 40, no. 1, pp. 41-46, 2008.

[29] F. Filipponi and F. Mosca, "Animal models of fulminant hepatic failure: need to test liver support devices," Digestive and Liver Disease, vol. 33, no. 7, pp. 607-613, 2001.
[30] G. Defterevos, C. Nastos, A. Papalois et al., "Peritoneal albumin dialysis as a novel approach for liver support: study in a porcine model of acute hepatic failure," Artificial Organs, vol. 40, no. 8, pp. 755-764, 2016.

[31] R. G. Kristiansen, C. F. Rose, O.-M. Fuskevåg et al., "Lornithine phenylacetate reduces ammonia in pigs with acute liver failure through phenylacetylglycine formation: a novel ammonia-lowering pathway," American Journal of PhysiologyGastrointestinal and Liver Physiology, vol. 307, no. 10, pp. G1024G1031, 2014.

[32] S. Benoist, R. Sarkis, M. Baudrimont et al., "A reversible model of acute hepatic failure by temporary hepatic ischemia in the pig," Journal of Surgical Research, vol. 88, no. 2, pp. 63-69, 2000.

[33] G. H. de Groot, C. B. Reuvers, S. W. Schalm et al., "A reproducible model of acute hepatic failure by transient ischemia in the pig," Journal of Surgical Research, vol. 42, no. 1, pp. 92-100, 1987.

[34] M.-P. van de Kerkhove, R. Hoekstra, T. M. van Gulik, and R. A. Chamuleau, "Large animal models of fulminant hepatic failure in artificial and bioartificial liver support research," Biomaterials, vol. 25, no. 9, pp. 1613-1625, 2004.

[35] Y. Gao, N. Mu, X.-P. Xu, and Y. Wang, "Porcine acute liver failure model established by two-phase surgery and treated with hollow fiber bioartificial liver support system," World Journal of Gastroenterology, vol. 11, no. 35, pp. 5468-5474, 2005.

[36] P. Zhou, J. Xia, G. Guo et al., "A Macaca mulatta model of fulminant hepatic failure," World Journal of Gastroenterology, vol. 18, no. 5, pp. 435-444, 2012. 


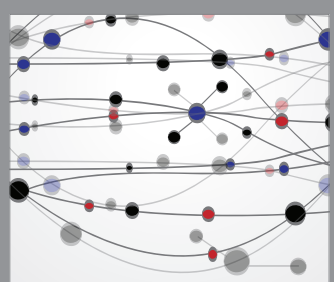

The Scientific World Journal
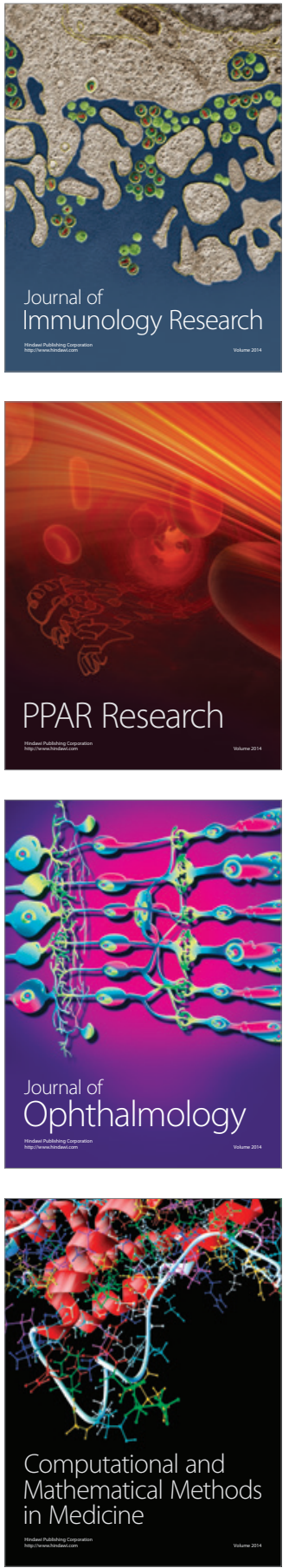

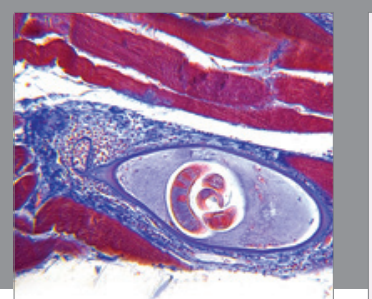

Gastroenterology Research and Practice

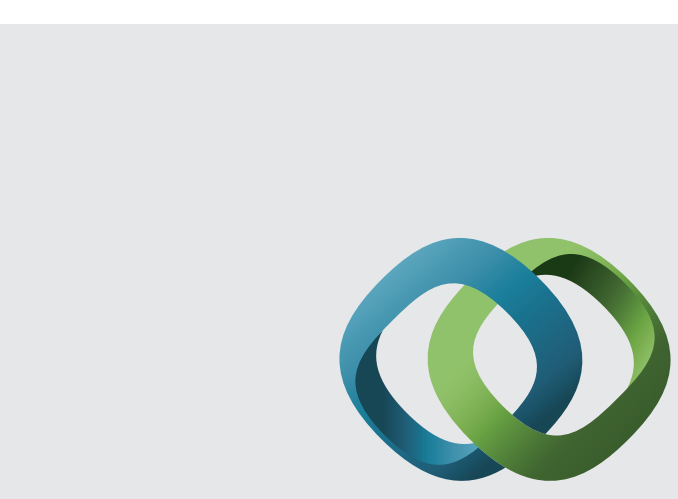

\section{Hindawi}

Submit your manuscripts at

http://www.hindawi.com
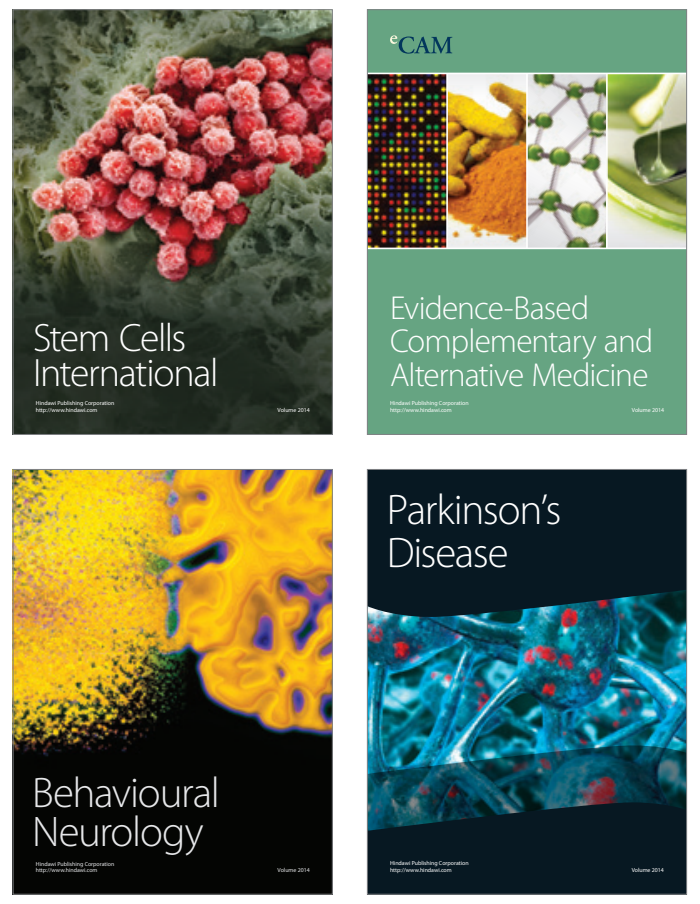
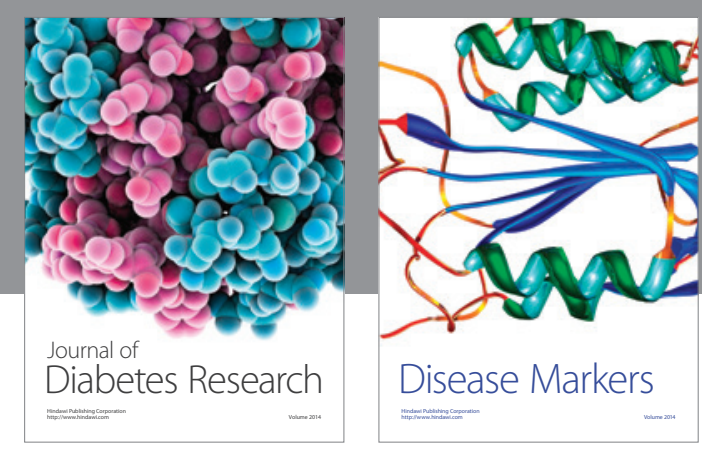

Disease Markers
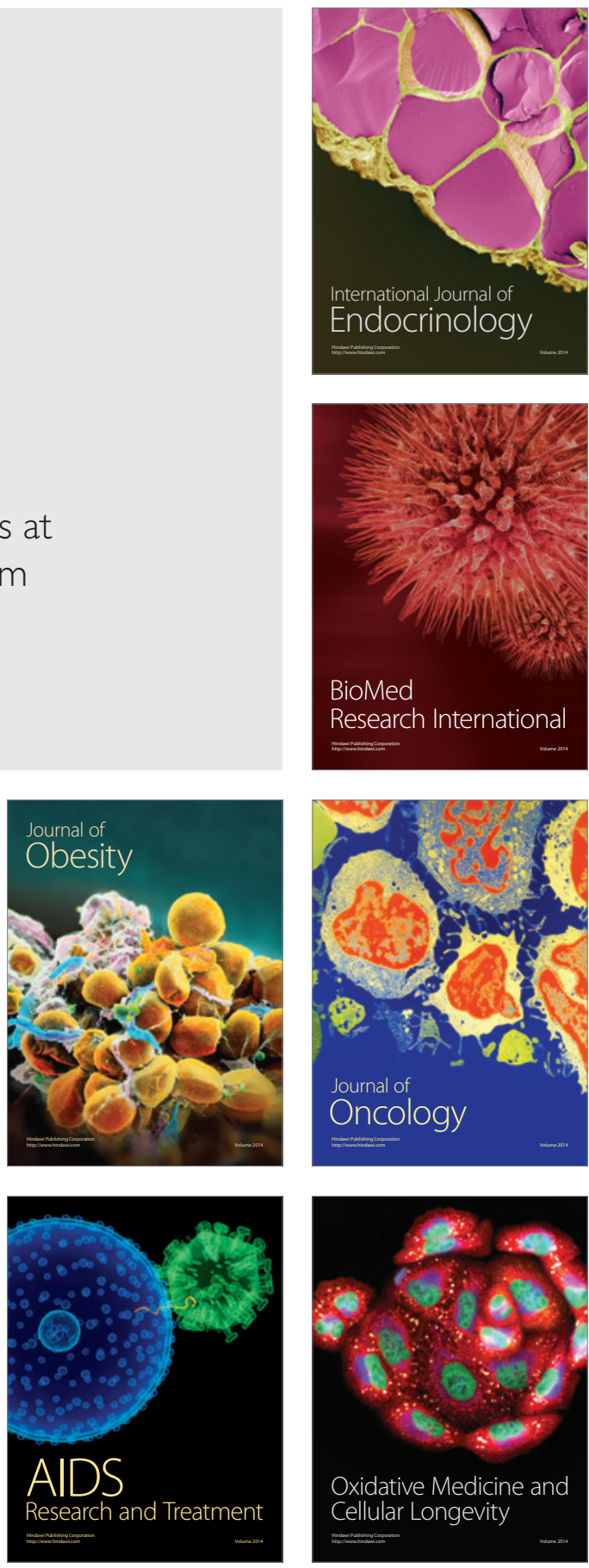